九州大学学術情報リポジトリ

Kyushu University Institutional Repository

\title{
Characterization of a cloned chromosomal fragment affecting differentiation in Streptomyces azureus ATCC14921
}

\section{Nishiyama, Takashi}

Laboratory of Microbial Genetics Technology, Department of Genetic Resources Technology, Division of Agriculture, Graduate School, Kyushu University

\section{Kamachi, Yasuharu}

Laboratory of Microbial Genetics Technology, Department of Genetic Resources Technology, Division of Bioresource and Bioenvironmental Sciences, Graduate School, Kyushu University

Yokoyama, Eiji

Laboratory of Microbial Genetics, Division of Applied Genetic Resources, Department of Genetic Resources Technology, Faculty of Agriculture, Graduate School, Kyushu University

Doi, Katsumi

Laboratory of Microbial Genetics, Division of Applied Genetic Resources, Department of Genetic Resources Technology, Faculty of Agriculture, Graduate School, Kyushu University

他

https://doi.org/10.5109/24373

出版情報：九州大学大学院農学研究院紀要. 45 (1)，pp.225-236，2000-11. Kyushu University バージョン：

権利関係 : 


\title{
Characterization of a cloned chromosomal fragment affecting differentiation in Streptomyces azureus ATCC14921
}

\author{
Takashi Nishiyama*, Yasuharu Kamachi**, Eiji Yokoyama, \\ Katsumi Doi and Seiya Ogata***
}

\author{
Laboratory of Microbial Genetics, Division of Applied Genetic Resources, \\ Department of Genetic Resources Technology, Faculty of Agriculture, \\ Graduate School, Kyushu University, Higashi-ku, Fukuoka 812-8581, Japan \\ (Received July 31, 2000 and accepted August 18, 2000)
}

\begin{abstract}
We characterized a transformant BalA1 of thiostrepton-producing strain Streptomyces azureus ATCC14921, which carries an approximately $2.5 \mathrm{~kb}$ chromosomal DNA fragment in high-copy number plasmid. While strain BalA1 formed little aerial hyphae, its morphological defect was restored by cultivation with $S$. azureus, $S$. laurentii and $S$. coelicolor. Strain BalAl strongly inhibited the growth of $B$. subtilis more so than its parent strain, and also inhibited the development of its parent and some Streptomyces strains with thiostrepton resistance. Furthermore, it induced $S$. coelicolor A3(2) to produce undecylprodgiosin at an early stage of growth, and to increase in the amount of actinorhodin production. The $2.5 \mathrm{~kb}$ fragment contained two orfs, orf1 and truncated orf2. The deduced products were somewhat similar to phosphoserine phosphatase-like protein and $\mathrm{N}$-terminal region of $\mathrm{MinD}$-like protein, respectively. The individual function of orf 1 or jointed function of both orfs seems to induce particular phenotypes or properties in strain BalA1.
\end{abstract}

\section{INTRODUCTION}

Streptomycetes undergo a complex cycle of morphological and physiological differentiation resembling that of filamentous fungi. A spore germinates to form substrate mycelium, aerial hyphae rise from the colony surface, and aerial hyphae septate into chains of pigmented spores. Morphological and physiological differentiation of Streptomyces are temporally coordinated (Champness and Chater, 1994), suggesting the existence of shared global regulatory elements for differentiation. Genes that promote morphological and physiological differentiation have been identified and characterized, include bld, whi, $a b a, a b s$ and $a f s$ (Aceti and Champness, 1998; Chater, 1998; Fernandez-Moreno et al., 1992; Umeyama et al., 1999). By cloning of regulatory sequences on high-copy-number plasmids, novel regulators for secondary metabolism and cellular differentiation have been detected and characterized (Scheu et al., 1997; Ueda et al., 1999). These reports encourage the use of high-copy-number plasmid to screen for potential developmental regulatory genes or sequences.

We isolated a $2.5 \mathrm{~kb}$ chromosomal DNA fragment from thiostrepton-producing Streptomyces azureus, using a high-copy-number plasmid. In this report, we char-

\footnotetext{
* Laboratory of Microbial Genetics Technology, Department of Genetic Resources Technology Division of Agriculture, Graduate School, Kyushu University

** Laboratory of Microbial Genetics Technology, Department of Genetic Resources Technology, Division of Bioresource and Bioenvironmental Sciences, Graduate School, Kyushu University

*** Corresponding author (E-mail: ogata@agr.kyushu-u.ac.jp)
} 
acterized the transformant and determined the sequence of the $2.5 \mathrm{~kb}$ fragment.

\section{MATERIALS AND METHODS}

\section{Bacterial strains, plasmids and growth conditions}

Streptomyces azureus PK100C, a plasmid-cured derivative of thiostrepton producing $S$. azureus ATCC14921 named as PK0, was earlier isolated in our laboratory (Miyoshi et al., 1986). Streptomyces coelicolor A3(2), its pIJ702 (Katz et al., 1983) carrying derivative and another thiostrepton-producing strain Streptomyces laurentii ATCC31255 were used as indicators in antimicrobial activity tests. Plasmid pMCP5, that was constructed by insertion of a kanamycin resistance gene from Streptomyces kanamyceticus ISP5500 into the BglII site of pIJ702 (Nakano et al., 1984), was kindly provided by H. Ogawara (Meiji Pharmaceutical University, Japan). Streptomyces cultures were grown in Bennett's or Rye media (Ogata et al., 1981). Solid media were prepared by addition of $1.5 \%$ agar (Difco). Thiostrepton and kanamycin were added at a concentration of $25 \mu \mathrm{g} \mathrm{ml}^{-1}$, as required.

Bacillus subtilis ATCC6633 was used as an indicator of antimicrobial activity. Solid cultures were prepared by mixing spores into autoclaved LB medium containing $1.5 \%$ agar at about $60^{\circ} \mathrm{C}$, then incubated at $37^{\circ} \mathrm{C}$.

E. coli strain JM109, host for pUC19 and pUC119, was grown at $37^{\circ} \mathrm{C}$ in $\mathrm{LB}$ medium supplemented with $50 \mu \mathrm{g} \mathrm{ml}^{-1}$ ampicillin.

Other bacterial strains used in this work are described in notes and figure legends, and all were grown under suitable conditions and appropriate media.

\section{General recombinant DNA techniques}

Transformation, plasmid isolation and DNA manipulation of Streptomyces and $E$. coli were done, as described (Hopwood et al., 1985). Restriction endonucleases and other modifying enzymes were purchased from TOYOBO (Japan). DNA sequence was determined using an ALFexpress automated sequencer and Thermo Sequenase fluorescent labelled primer cycle sequencing kits with 7-deaza-dGTP (Amersham Pharmacia Biotech). Computer analysis was made using GENETYX-MAC program (Software Development Co. Ltd., Japan). Similar nucleotide or amino acid sequences were identified using BLAST.

\section{Shotgun cloning}

Chromosomal DNA prepared from $S$. azureus $\mathrm{PK100C}$ was digested with $K p n \mathrm{I}$ and ligated into the KpnI site of pMCP5. The ligation mixture was used to transform $S$. azureus PK100C, and kanamycin-resistant transformants were selected (Tomura et al., 1993). A colony lacking aerial hyphae was cultured in liquid Bennett's medium. The recombinant plasmid was extracted and re-transformed strain PK100C to confirm that the aerial hyphae-negative phenotype was the result of cloned DNA fragment.

\section{Physiological activity}

Antimicrobial activity was tested using the agar piece method. Pre-cultivated $S$. azureus PK100C and BalA1 in liquid Bennett's media were spreaded to Rye plates $\left(10^{6}\right.$ 
colony-forming units per plate). These solid cultures sampled using a cork-borer were transferred onto an indicator lawn of $B$. subtilis ATCC6633. Further physiological activity was observed by streaking BalA1 proximate to indicator strains.

\section{Southern hybridization}

Chromosomal DNAs of Streptomyces strains, prepared as described (Hopwood et al., 1985), were digested with KpnI. After agarose gel electrophoresis, the DNA fragments were transferred onto a PROTRAN BA85 nitrocellulose membrane (Schleicher \& Schuell, Germany). The $2.5 \mathrm{~kb}$ fragment used as a probe was labeled with DIG DNA Labeling and Detection kit (Roche Diagnostics). Hybridization was carried out following the manufacturer's instructions.

\section{RESULTS}

\section{Isolation of transformant BalA1}

One colony impaired in aerial mycelium-formation was isolated out of approximately 30,000 shotgun-cloned transformants (Fig. 1, right). This transformant carried a plasmid pSAB931 which we constructed by insertion of an approximately $2.5 \mathrm{~kb}$ chromosomal DNA fragment into the KpnI site of pMCP5. The aerial mycelium-negative phenotype was confirmed by re-introduction of pSAB931 into $S$. azureus PK100C, and the transformant was named BalA1. A transformant with only vector pMCP5 did not show the aerial hyphae-negative phenotype and the following properties.

It is well known that medium pH pleiotropically affects morphological development of

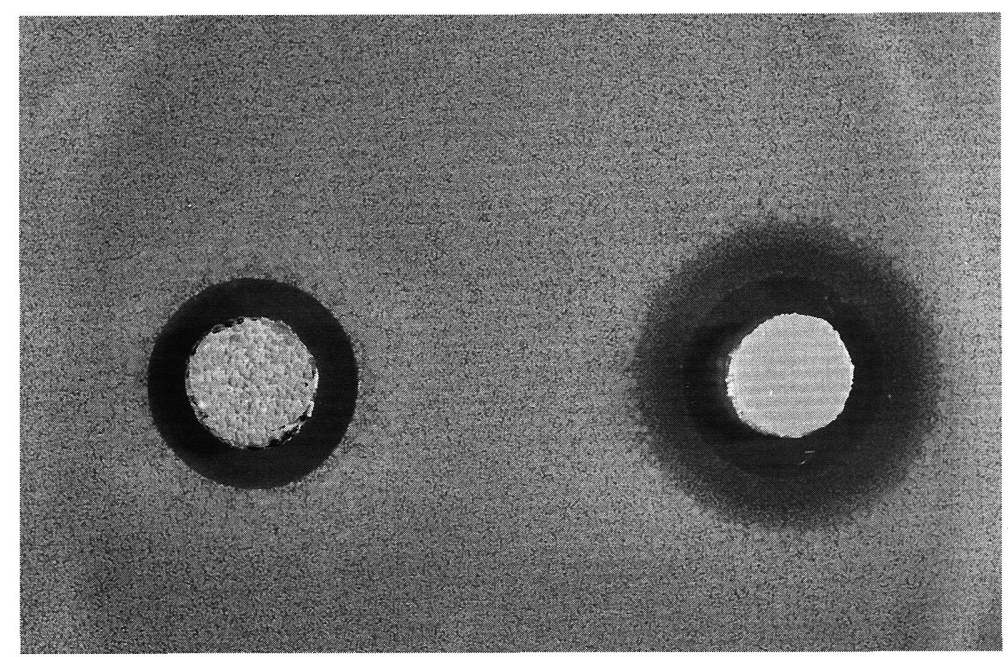

Fig. 1. Growth inhibition toward B. subtilis ATCC6633 by S. azureus BalA1 and its parent PK100C.

After seven days of growth, agar pieces of strains BalAl (right) and PK100C (left) were transferred onto a B. subtilis seeded plate, and incubated at $37^{\circ} \mathrm{C}$ for $1 \mathrm{~d}$. 
Streptomyces (Süsstrunk et al., 1998). However, the phenotype of strain BalA1 was not suppressed by neutralizing buffer (data not shown).

\section{Physiological activity}

As shown in Fig. 1, a piece of solid culture of strain BalA1 not only formed a clear growth-inhibitory zone toward B. subtilis ATCC6633 as large as that of parent strain PK100C, but also formed a turbid area outside of the clear zone (Fig. 1, right). Strain BalA1 inhibited the morphological development of thiostrepton resistant Streptomyces strains, including its parent strain PK100C (Fig. 2) and another thiostrepton producing strain S. laurentii ATCC31255 (Fig. 3, A, B). And strain BalA1 also inhibited morphological development of PK0, wild-type strain of $S$. azureus, and S. coelicolor A3(2) transformed with pIJ702 (Fig. 3, A; Table 1), which carried the thiostrepton resistance gene of $S$. azureus (Katz et al., 1983). We interpreted these phenomena to mean that strain BalA1 secreted some bioactive substance(s) in addition to thiostrepton.

During growth with $S$. azureus and S. laurentii ATCC31255, formation of aerial hyphae and spores of strain BalA1 was restored (Fig. 3, A, B). Weaker restoration was also observed with Streptomyces griseus JCM4681 and Streptomyces fradiae JCM4133 (Table 1). These restorations may suggest that strain BalA1 lacks an important substance or regulator for initiating morphological development, or that a developmental inhibitor caused by pSAB931 is inactivated.

Furthermore, strain BalA1 induced S. coelicolor A3(2) early production of red

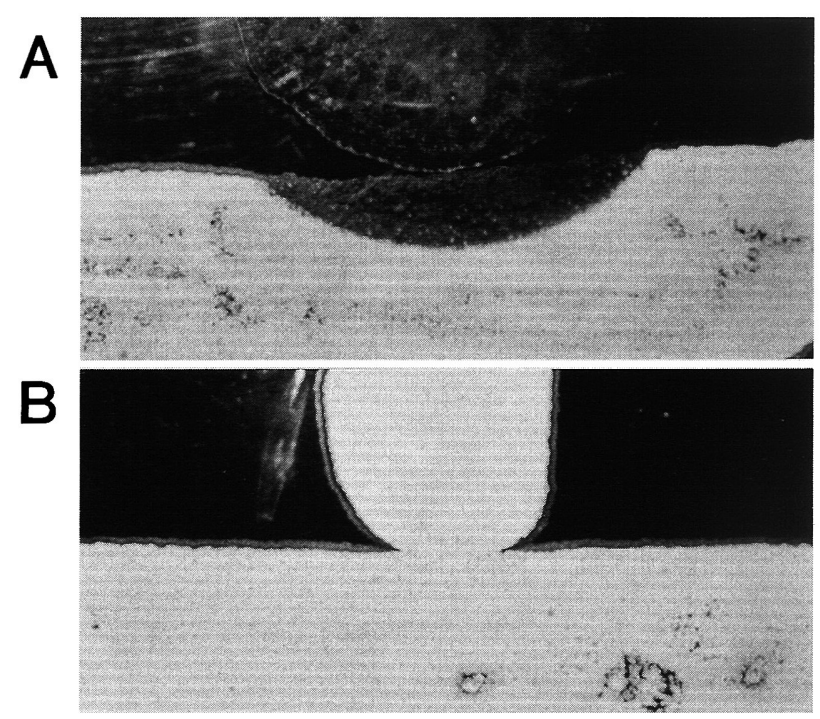

Fig. 2. Physiological activities of strain BalA1.

Strain BalA1 and its parent PK100C were cultivated proximately for $3 \mathrm{~d}$. Strain BalAl (A, upper half) inhibited morphological differentiation of PK100C (A, lower half), but not so PK100C carrying the vector pMCP5 (B, upper half). 

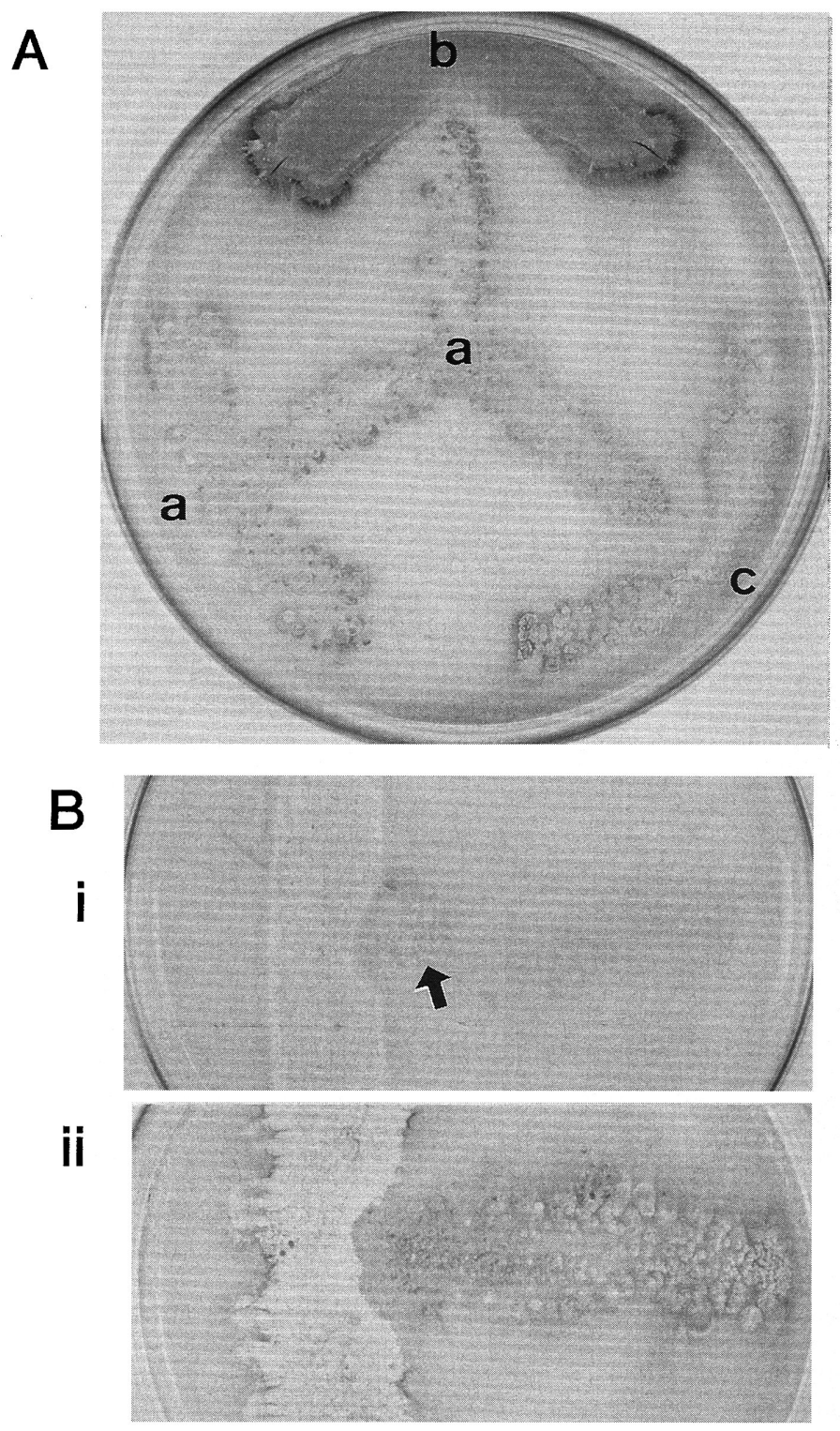

Fig. 3. Inhibition of morphological development toward $S$. coelicolor and $S$. laurentii by strain BalA1, and restoration of morphological differentiation of strain BalA1 by them.

A: Strain BalA1 (a), S. coelicolor A3(2) carrying pIJ702 (b) and thiostrepton-producing strain $S$. laurentii ATCC31255 (c) were cultivated proximately for $7 \mathrm{~d}$. Strain BalA1 inhibited morphological development of thiostrepton resistant strains (b, c). Needless to say, no inhibition was observed between strain BalA1s (a). Aerial mycelia and spores of strain BalA1 was restored on the entire lawn.

B: Strain BalA1 (right) and S. laurentii ATCC31255 (left) were cultivated proximately for $3 \mathrm{~d}$ (Bi). The aerial mycelia of strain BalA1 appeared at the area neighboring lawn of $S$. laurentii (Bi, arrow). After $7 \mathrm{~d}$ cultivation, aerial mycelia and spores of strain BalA1 were observed on the entire lawn (Bii). 
Table 1. Physiological activities of $S$. azureus BalA1

Thiostrepton resistant strains which were inhibited their morphological differentiation by strain BalA1
Strains which restore the formation of aerial hyphae and spores of strain BalA1 $\dagger$
S. azureus $\mathrm{PK} 0 *$
S. azureus PK0*
S. azureus PK100C
S. azureus PK100C
S. coelicolor A3(2) with pIJ702
S. coelicolor A3(2) with pIJ702
S. laurentii ATCC31255
$S$. griseus JCM4681
S. fradiae JCM 4133
S. laurentii ATCC31255

* S. azureus ATCC14921

$\dagger$ Restorations were observed in the same way as Fig. 3.

\section{A}

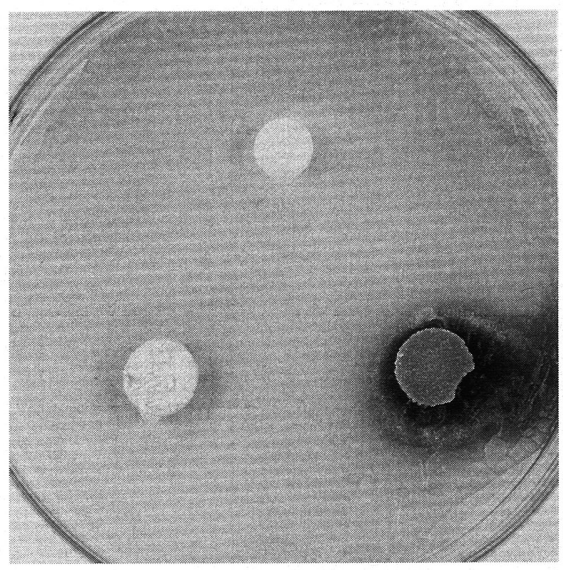

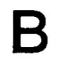

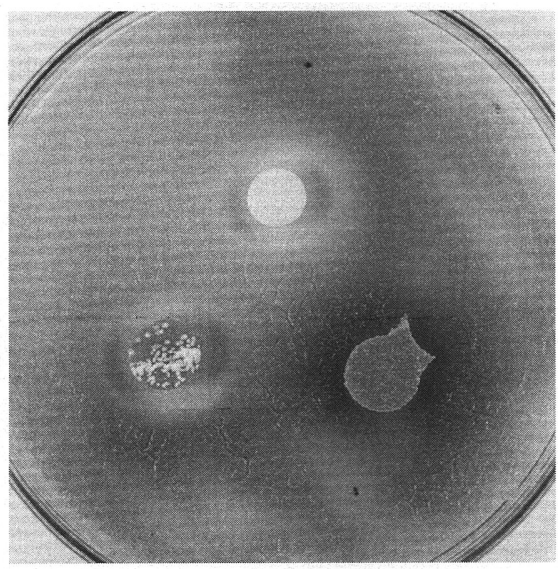

Fig. 4. Influence of strain BalAl on pigmentary antibiotics productions of $S$. coelicolor $A 3(2)$. After seven days of growth, agar pieces of strains BalAl were transferred onto seeded plates of $S$. coelicolor, and incubated for more than $2 \mathrm{~d}$.

A: Strain BalA1 induced early production of undecylprodgiosin at $2 \mathrm{~d}$ or less (lower right).

B: Strain BalA1 had barely induced early production of actinorhodin, but increased in the amount after $2 \mathrm{~d}$ (lower right).

Strain PK100C (lower left) and paper disk (8m in diameter) containing $10 \mu \mathrm{g}$ thiostrepton (upper center) did not affect the antibiotics productions.

pigmentary antibiotic undecylprodgiosin (Fig. 4, A). The same effect was obtained with the addition of supernatant of liquid culture of strain BalA1 (data not shown). Strain BalA1 did not induced early production of actinorhodin, blue pigmentary antibiotic of $S$. coelicolor, but increased in the amount (Fig. 4, B). The parent strain PK100C and its 
antibiotic thiostrepton did not affect pigment production of $S$. coelicolor A3(2). These results suggested that strain BalA1 probably produced inducer(s) or regulator(s) for production of secondary metabolites of Streptomyces.

\section{Nucleotide sequence}

The nucleotide sequence of the cloned $2506 \mathrm{bp}$ fragment was determined. FRAME analysis (Bibb et al., 1984) by GENETYX-MAC revealed a complete 834 bp orf (orf1) and an incomplete $541 \mathrm{bp}$ orf (orf2) in this region (Fig. 5). The entire nucleotide sequence of the fragment was deposited in DDBJ under accession no. AB004855.

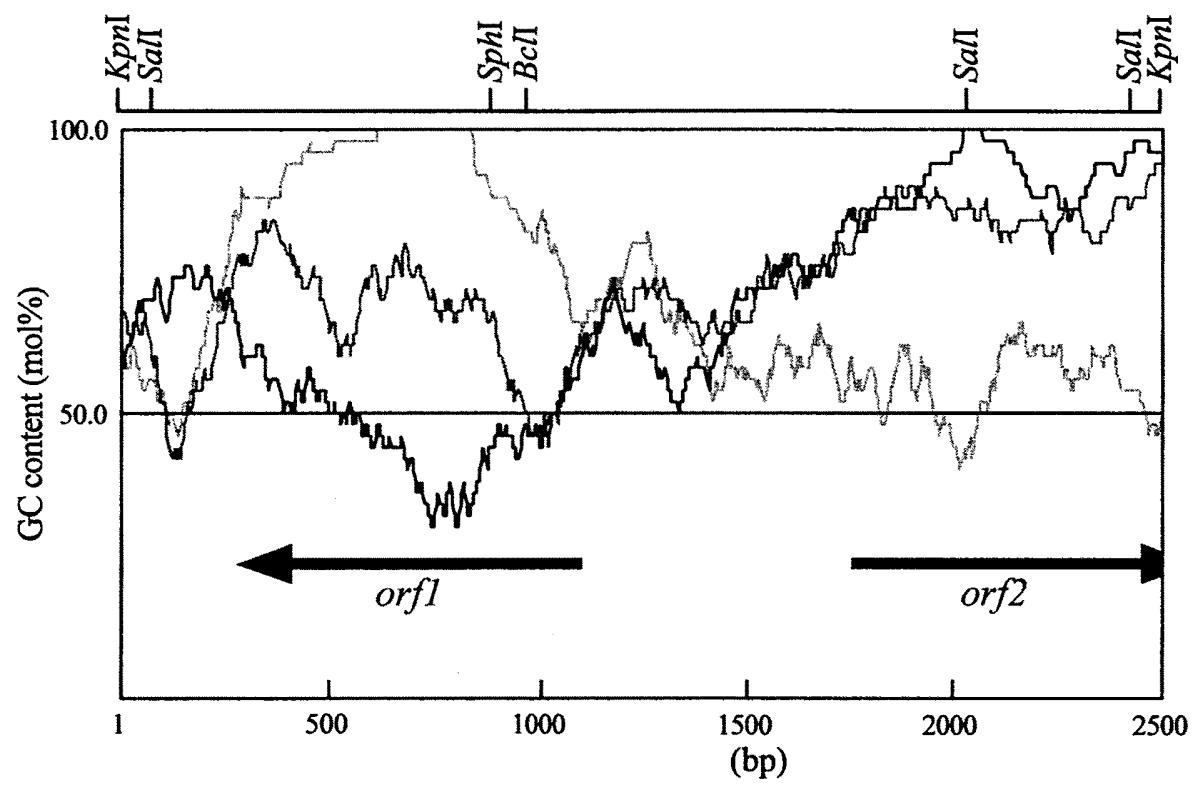

Fig. 5. Restriction map with $\mathrm{GC}-$ plot and nucleotide sequence of the cloned fragment. The FRAME analysis was carried out with window size $50 \mathrm{nt}$.

\section{Homology search}

The nucleotide sequence and deduced amino acid sequences of orf 1 and orf 2 were compared with sequences in databases. There was no similar gene, protein or sequence relating to the differentiation in Streptomyces. Orf1 showed similarity to phosphoserine phosphatase-like protein of Mycobacterium leprae (Fig. 6, A) (accession no. U00018, $37 \%$ identity and $73 \%$ similarity in the overlapped region), and others. Orf2 showed a low level of similarity to MinD, septum site determining protein, of B. subtilis (Fig. 6, B) (accession no. AL009126, 14\% identity and 58\% similarity in the overlapped region) (Varley and Stewart, 1992) and MinD-like proteins of several species. Although MinD protein was seen to have a mononucleotide binding motif and ATPase activity (de Boer et $a l ., 1991$ ), Orf2 lacked the motif replacing the most important lysine residue with alanine (Saraste et al., 1990). 


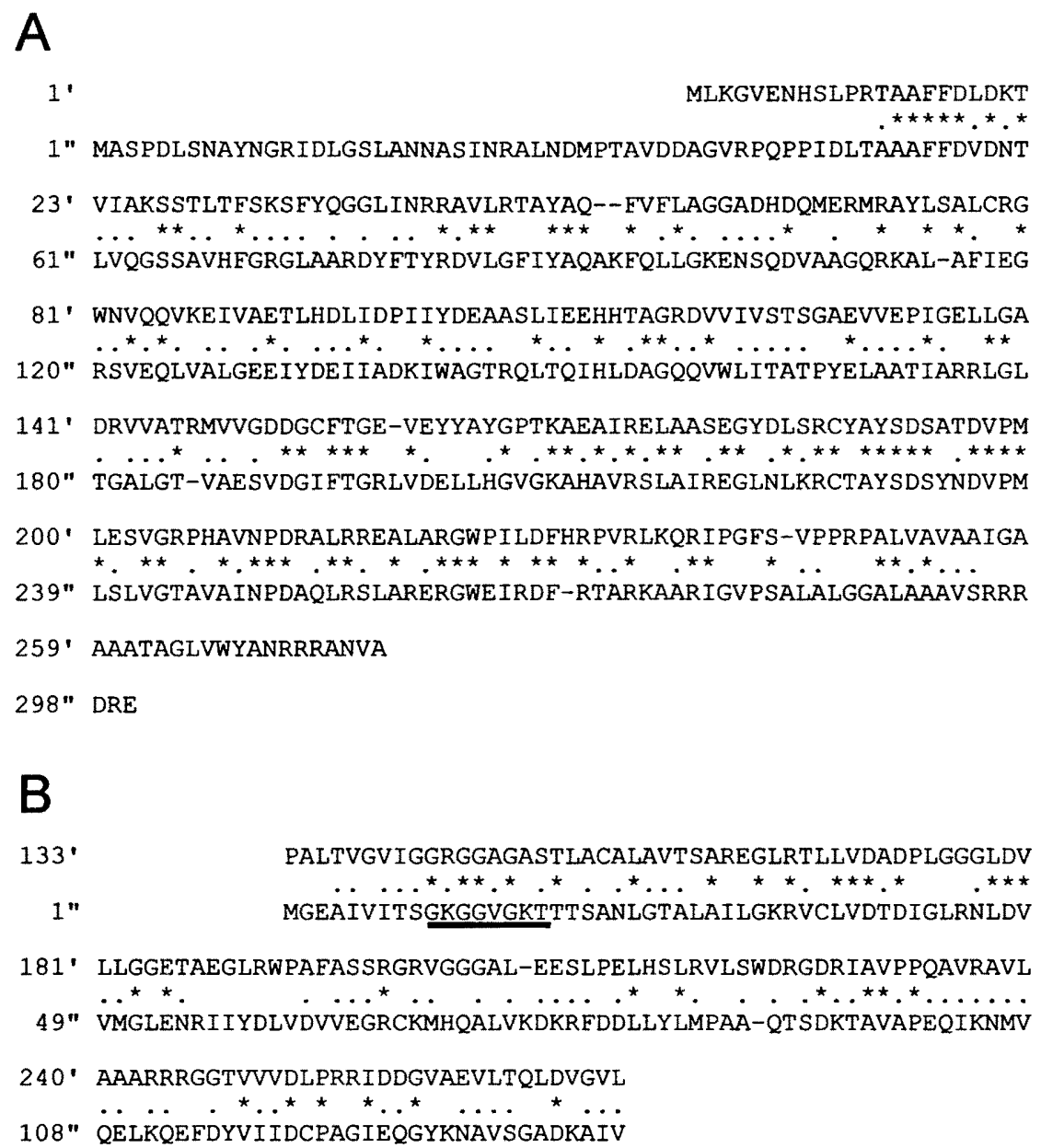

Fig. 6. Comparison of amino acid sequences between Orfs in the $2.5 \mathrm{~kb}$ fragment and registered sequences in database.

A: Orfl (upper line) and phosphoserine phosphatase-like protein of $M$. leprae (lower line). B: Orf2 (upper line) and MinD protein of $B$. subtilis (lower line).

Identical and similar residues are designated with an asterisk and a dot, respectively. Underline indicates the mononucleotide binding motif (P-loop: GXXXXGK[TS]).

\section{Southern blot analysis}

To search for similar sequences, Southern hybridization was done using the $2.5 \mathrm{~kb}$ fragment as a probe against total DNAs from many kinds of bacteria. Fig. 7 shows hybridizing bands with Streptomyces lividans TK21, S. lividans TK24, S. laurentii ATCC31255, S. coelicolor JCM4357, S. griseus JCM4681 and S. griseus JCM4319. The same results were obtained with other Streptomyces strains, but not from Gordonia 


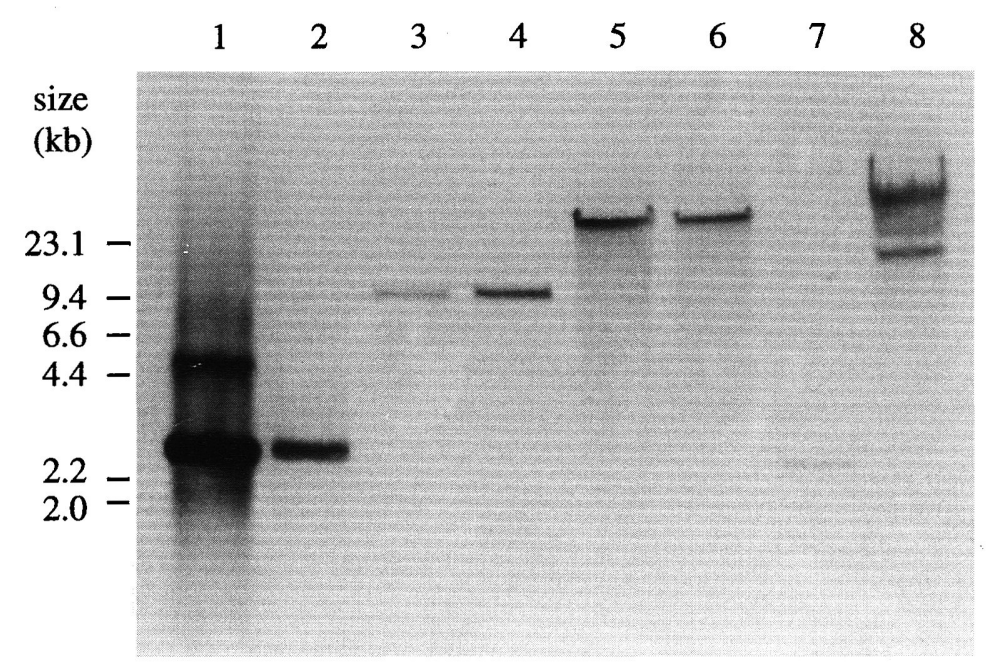

Fig. 7. Southern blot of several Streptomyces strains.

The probe was the DIG-labelled $2.5 \mathrm{~kb}$ fragment. All DNAs were digested with KpnI. Lanes: 1 , the $2.5 \mathrm{~kb}$ fragment carried on the plasmid pMCP5; 2, S. azureus PK100C; 3, Streptomyces lividans TK21; 4, S. lividans TK24; 5, S. laurentii ATCC31255; 6, S. coelicolor JCM4357; 7, S. griseus JCM4681; 8, S. griseus JCM4319.

amarae JCM3171, Nocardia farcinica JCM3088, Rhodococcus sp JCM3376, B. subtilis ATCC6633 and E. coli JM109 (Table 2). These results indicate that regions similar to the $2.5 \mathrm{~kb}$ fragment are widely distributed among Streptomyces species. Recently we found a sequence (registered as highly homologous to the $2.5 \mathrm{~kb}$ fragment detected in this work) in a database of Streptomyces coelicolor A3(2) Genome Project (SCH5.19-20c region of $S$. coelicolor cosmid H5, accession no. AL035636, Nucleotide identity is $81 \%$ in overlapped region). These results imply that regulation systems similar to strain BalA1 play important global roles in the early differentiation of Streptomyces.

\section{DISCUSSION}

Strain BalA1, which carried with high-copy-number plasmid including an approximately $2.5 \mathrm{~kb}$ fragment, formed little aerial mycelium, yet this morphological defect was restored by growth with Streptomyces strains, and this restoration led to the formation of spores. Strain BalA1 produced an unidentified antimicrobial substance in addition to thiostrepton, and induced early production of red pigmentary antibiotic undecylprodgiosin in $S$. coelicolor. These phenomena were reminiscent of regulators such as A-factor, which plays a key role as regulator to activate signal transduction pathways for differentiation (Ohnishi et al., 1999). The repression or de-repression caused by the unidentified regulatory element may be responsible for the particular phenotype of strain 
Table 2. Summarized result of Southern hybridization using the $2.5 \mathrm{~kb}$ fragment as a probe

\begin{tabular}{lc}
\hline \multicolumn{1}{c}{ Strain } & Hybridization \\
\hline Streptomyces azureus PK0 & + \\
S. azureus PK100C & + \\
S. achromogenes JCM4121 & + \\
S. antibioticus JCM4007 & + \\
S. coelicolor JCM4357 & + \\
S. coelicolor A3(2) & + \\
S. coerulescens JCM4360 & + \\
S. cyaneus JCM4220 & + \\
S. fradiae JCM4133 & + \\
S. glaucescens JCM4377 & + \\
S. griseus JCM2926 & + \\
S. griseus JCM4681 & + \\
S. hawaiiensis JCM4172 & + \\
S. lactamdurans JCM4912 & + \\
S. laurentii ATCC31255 & + \\
S. lavendulae JCM4055 & + \\
S. lividans TK21 & + \\
S. lividans TK24 & + \\
S. sioyaensis JCM4418 & + \\
Gordonia amarae JCM3171 & - \\
Nocardia farcinica JCM3088 & - \\
Rhodococcus sp JCM3376 & - \\
Bacillus subtilis ATCC6633 & - \\
Escherichia coli JM109 & - \\
\hline . & + \\
\hline
\end{tabular}

+ , Hybridized with the $2.5 \mathrm{~kb}$ fragment; - , No hybridization

Total DNA of these strains were digested with KpnI and hybridized with the $2.5 \mathrm{~kb}$ fragment.

BalA1, however, the A-factor did not affect the properties of strain BalA1 (data not shown).

The $2.5 \mathrm{~kb}$ fragment is expected to influence the regulation systems of morphological differentiation and secondary metabolite, and/or to contain some regulatory element(s) involved in differentiation. It has been reported that putative regulators inserted in high-copy-number plasmids influenced a carbon-source-dependent morphological defect in $S$. griseus and secondary metabolite production of $S$. lividans (Scheu et al., 1997; Ueda et al., 1999). The $2.5 \mathrm{~kb}$ fragment showed no similarity to previously characterized genes or to sequences for differentiation in Streptomyces, judging from the sequence of the $2.5 \mathrm{~kb}$ fragment and two orfs. However, these orfs showed little similarity to phosphoserine phosphatase-like proteins and MinD (and MinD-like) proteins. Phosphoserine phosphatase and MinD are involved in serine biosynthesis and inhibition of septum formation at potential division sites, respectively (Hu and Lutkenhaus, 1999; Neuwald and Stauffer, 1985). Thus, the orfs may influence serine concentration or cell division in mycelium. These quantitative or qualitative changes are possibly key elements for differentiation in Streptomyces, or the orfs themselves may be novel regulatory 
elements. Thus, functional analysis of orf1 and orf2 may contribute greatly to understanding of mechanisms of differentiation in Streptomyces. Detailed characteristics of orf1 and orf 2 will be reported in ongoing studies.

\section{ACKNOWLEDGEMENTS}

This work was supported by a Grant-in-Aid for Scientific Research (C, no. 06660115) from the Ministry of Education, Science, Sports and Culture of Japan and by the Suzuki Fellowship Foundation (Tokyo). We are grateful to M. Ohara for helpful comments and advice on the manuscript.

\section{REFERENCES}

Aceti, D. J. and W. C. Champness 1998 Transcriptional regulation of Streptomyces coelicolor pathway-specific antibiotic regulators by the $a b s A$ and $a b s B$ loci. J. Bacteriol, 180: 3100-3106.

Bibb, M. J., P. R. Findlay and M. W. Johnson 1984 The relationship between base composition and codon usage in bacterial genes and its use for the simple and reliable identification of protein-coding sequences. Gene, 30: 157-166.

Champness, W. C. and K. F. Chater 1994 Regulation and integration of antibiotic production and morphological differentiation in Streptomyces spp. In: "Regulation of bacterial development" ed. by Piggot, P. J., Moran, C. P. and Youngman, P., American Society for Microbiology, Washington, DC, pp. 61-93.

Chater, K. F. 1998 Taking a genetic scalpel to the Streptomyces colony. Microbiology, 144: 1465-1478.

de Boer, P. A. J., R. E. Crossley, A. R. Hand and L. I. Rothfield 1991 The MinD protein is a membrane ATPase required for the correct placement of the Escherichia coli division site. EMBO J., 10: $4371-4380$.

Fernandez-Moreno, M. A., A. J. Martin-Triana, E. Martinez, J. Niemi, H. M. Kieser, D. A. Hopwood and F. Malpartida $1992 a b a A$, a new pleiotropic regulatory locus for antibiotic production in Streptomyces coelicolor. J. Bacteriol., 174: 2958-2967.

Hopwood, D. A., M. J. Bibb, K. F. Chater, T. Kieser, C. J. Bruton, H. M. Kieser, D. J. Lydiate, C. P. Smith, M. J. Ward and H. Schrempf 1985 Genetic manipulation of Streptomyces: a laboratory manual. John Innes Foundation, Norwich (England)

$\mathrm{Hu}, \mathrm{Z}$. and J. Lutkenhaus 1999 Topological regulation of cell division in Escherichia coli involves rapid pole to pole oscillation of the division inhibitor MinC under the control of MinD and MinE. Mol. Microbiol., 34: 82--90.

Katz, E., C. J. Thompson and D. A. Hopwood 1983 Cloning and expression of the tyrosinase gene from Streptomyces antibioticus in Streptomyces lividans. J. Gen. Microbiol., 129: 2703-2714.

Miyoshi, Y. K., S. Ogata and S. Hayashida 1986 Multicopy derivative of pock-forming plasmid pSA1 in Streptomyces azureus. J. Bacteriol., 168: 452-454.

Nakano, M. M., H. Mashiko and H. Ogawara 1984 Cloning of the kanamycin resistance gene from a kanamycin-producing Streptomyces species. J. Bacteriol., 157: 79-83.

Neuwald, A. F. and G. V. Stauffer 1985 DNA sequence and characterization of the Escherichia coli serB gene. Nucleic. Acids. Res., 13: 7025-7039.

Ogata, S., S. Yoshino, H. Suenaga, K. Aoyama, N. Kitajima and S. Hayashida 1981 Specific lysogenicity in Streptomyces azureus. Appl. Environ. Microbiol, 42: 135-141.

Ohnishi, Y., S. Kameyama, H. Onaka and S. Horinouchi 1999 The A-factor regulatory cascade leading to streptomycin biosynthesis in Streptomyces griseus: identification of a target gene of the A-factor receptor. Mol. Microbiol., 34: 102-111

Saraste, M., Sibbald, P. R. and A. Wittinghofer 1990 The P-loop - a common motif in ATP- and GTP-binding proteins. Trends. Biochem. Sci., 15: 430-434.

Scheu, A. K., E. Martinez, J. Soliveri and F. Malpartida 1997 abaB, a putative regulator for secondary metabolism in Streptomyces. FEMS Microbiol. Lett., 147: 29-36. 
Süsstrunk, U., J. Pidoux, S. Taubert, A. Ullmann and C. J. Thompson 1998 Pleiotropic effects of cAMP on germination, antibiotic biosynthesis and morphological development in Streptomyces coelicolor. Mol. Microbiol., 30: 33-46.

Tomura, T., H. Kishino, K. Doi, T. Hara, S. Kuhara and S. Ogata 1993 Sporulation-inhibitory gene in pock-forming plasmid pSA1.1 of Streptomyces azureus. Biosci. Biotechnol. Biochem., 57: 438 443.

Ueda, K., K. Matsuda, H. Takano and T. Beppu 1999 A putative regulatory element for carbon-source-dependent differentiation in Streptomyces griseus. Microbiology, 145: 2265-2271.

Umeyama, T., P. C. Lee, K. Ueda and S. Horinouchi 1999 An AfsK/AfsR system involved in the response of aerial mycelium formation to glucose in Streptomyces griseus. Microbiology, 145: 2281-2292.

Varley, A. W, and G. C. Stewart 1992 The divIVB region of the Bacillus subtilis chromosome encodes homologs of Escherichia coli septum placement (MinCD) and cell shape (MreBCD) determinants. $J$. Bacteriol., 174: 6729-6742. 